\title{
Dimensionality transition in submonolayer growth on carbon nanotubes
}

\author{
R. Delagrange, F. Tournus, L. Bardotti, J. M. Benoit, and O. Pierre-Louis \\ Institut Lumière Matière, UMR5306 Université Lyon 1-CNRS, Université de Lyon 69622 Villeurbanne, France
}

(Received 5 April 2013; revised manuscript received 27 December 2013; published 21 January 2014)

\begin{abstract}
We report on the transition from two-dimensional to one-dimensional (1D) behavior observed in submonolayer deposition on a tubular substrate. Using kinetic Monte Carlo simulations we show that the island density increases when the tube diameter decreases, approaching the 1D behavior. This increase is confirmed by experiments of FePt nanoparticle deposition on carbon nanotubes. Kinetic Monte Carlo simulations also indicate that the consequences of confinement sharply disappear when the perimeter of the tube is slightly larger than the distance between islands.
\end{abstract}

DOI: 10.1103/PhysRevB.89.035425

PACS number(s): 68.55.-a, 81.10.Aj

\section{INTRODUCTION}

Many nonequilibrium systems exhibit a critical behavior, usually belonging to a given universality class characterized by exponents [1]. However, critical behaviors depends strongly on dimensionality [1], and the transition between different dimensionalities is still poorly explored, except for a few examples such as domain walls in ferromagnetic nanowires [2]. In this paper we propose a system; namely, submonolayer deposition on a tube, where this transition can be investigated. Submonolayer growth is a prototypical nonequilibrium system exhibiting critical behavior, which has been studied extensively both experimentally [3-6] and theoretically [7-13]. In this process, particles deposited on a substrate diffuse and aggregate to form islands. Standard scaling predictions describe the dependence of island-size distribution and the island density as a function of the coverage and the deposition rate. Much effort was also recently devoted to modeling the island-size distribution and the capture-zone distribution [13-16]. Theories and experiments of submonolayer growth are available both for two-dimensional (2D) and onedimensional (1D) systems. The majority of experiments has been performed on atomically flat crystal facets, corresponding to the $2 \mathrm{D}$ case $[9,11]$. Experiments have also been performed in the 1D case, a prominent example being adsorption on linear defects, such as atoms or fullerenes on atomic-step edges [4,17-21], or nanoparticles on graphite pleats [22,23]. In addition, some models have discussed the interplay between 2D and 1D effects in submonolayer growth due to the presence of atomic steps on facetted substrates [24].

Here we discuss the transition from 1D to 2D behavior in submonolayer growth with tubular substrate geometries. In a large 2D periodic system of size $L_{x} \times L_{y}$, the transition to $1 \mathrm{D}$ can be obtained by reducing the size $L_{x}$ down to the particle size (keeping a large $L_{y}$ ). Experimentally, such a variation of $L_{x}$ corresponds to performing growth on the surface of tubes with different diameters. The length $L_{x}$ then corresponds to the tube perimeter. In the following, we present kinetic Monte Carlo (KMC) simulations with various values of $L_{x}$. We focus on the limit of small coverages which corresponds to experiments, and where coalescence of different islands and island percolation in the direction $y$ along the tube are not observed. However, coalescence of each island with itself around the perimeter of the tube in the $x$ direction can be observed for small $L_{x}$. We find an increase of the island density when reducing the tube diameter. This result is confirmed by experiments with deposition of $\mathrm{FePt}$ nanoparticles on carbon nanotubes with diameters ranging from 5 to $10 \mathrm{~nm}$. Furthermore, a detailed analysis of the KMC results indicates a transition from a $2 \mathrm{D}$ to a $1 \mathrm{D}$ behavior. We find that the consequences of the confinement vanish sharply for distances larger than the typical distance between islands. In the following, we first present the KMC simulation results. Then we discuss the experiments.

\section{KINETIC MONTE CARLO MODEL}

We have performed KMC simulations to model the diffusion of nanoparticles and the subsequent formation of islands. Particles diffuse on a hexagonal lattice. The hopping rate of atoms with $n$ in-plane nearest neighbors is

$$
v_{n}=v \mathrm{e}^{-n J / k_{B} T},
$$

where $v$ is the hopping rate of particles without nearest neighbor, $J$ is the bond energy between nearest neighbors, $k_{B}$ is the Boltzmann constant, and $T$ is the temperature. In addition, we deposit particles at the rate $F$. In order to do so, a site is chosen randomly. If the site is free, we deposit a particle. When a particle is deposited on a site which is already occupied, we move it to a neighboring site which is free. If there is no free site in the nearest neighbors, we do not deposit the particle. The dynamics depends only on two dimensionless parameters: $R=v / F$ and $J / k_{B} T$.

We use periodic boundary conditions with $L_{x} \times L_{y}$ lattice sites. In order to study the influence of the confinement produced by a finite nanotube diameter, we perform simulations with various values of $L_{x}$. In the simulations, we keep a fixed total area $L_{x} \times L_{y}=150^{2}$.

\section{KINETIC MONTE CARLO RESULTS: ISLAND-SIZE DISTRIBUTIONS}

Submonolayer growth is known to exhibit universal scaling of the island-size distribution [7]. There are several regimes depending on the coverage $\theta$ [25]. Increasing $\theta$, one first finds a nucleation regime at low $\theta$ where new islands are nucleated. Then, at intermediate $\theta$, one finds the so-called aggregation regime where islands grow via sticking of new particles. In this regime islands do not coalesce with other islands, but for small tube perimeters $L_{x}$ islands can coalesce with themselves 


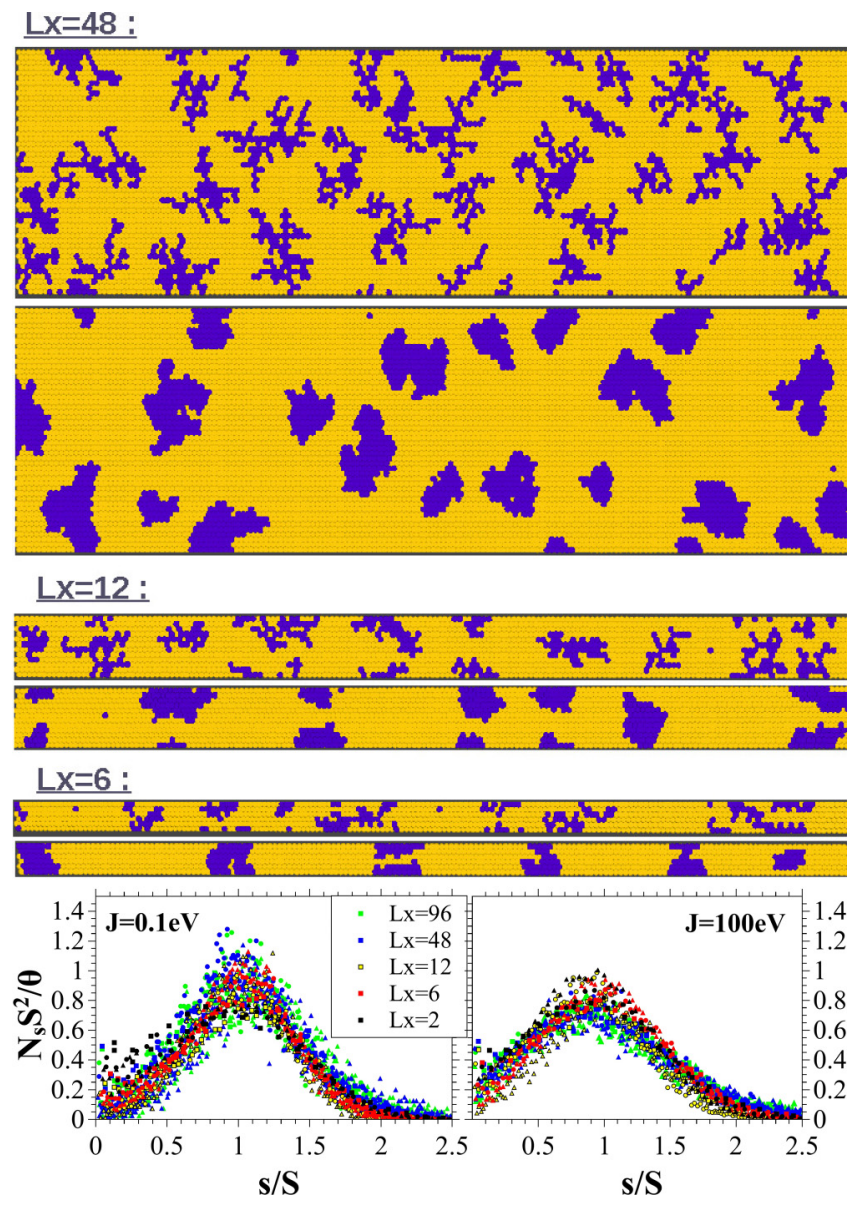

FIG. 1. (Color online) Kinetic Monte Carlo simulations of submonolayer deposition on tubes of various perimeters $L_{x}=48,12,6$ (the length $L_{y}$ is larger than the images). The tubes are unrolled for clarity. For each $L_{x}$, the upper panel corresponds to irreversible DLAlike aggregation at $J=100 \mathrm{eV}$, and the lower panel corresponds to the attachment-detachment regime, with $J=0.1 \mathrm{eV}$. The two graphs show the data collapse for the island-size distribution in the attachment-detachment and irreversible aggregation regimes with $R=10^{5}$. Symbols: $\mathbf{\square}, \boldsymbol{\bullet}, \boldsymbol{\Delta}$ for $\theta=0.1,0.2,0.3$, respectively.

around the tube as seen in Fig. 1 for $L_{x}=6$. Finally, at larger $\theta$, islands start to coalesce with other islands.

We focus on the aggregation regime [25], which describes the system behavior at intermediate $\theta$ (typically between 5\% and $30 \%$ ). We have verified the usual scaling of the density $N_{s}$ of islands of size $s$ :

$$
N_{s}=\frac{\theta}{S^{2}} f\left(\frac{s}{S}\right),
$$

where $f$ is a universal scaling function, and $S$ is the average island size. We have checked that this scaling law is observed in our simulations: for a given value of $J / k_{B} T$, the scaled island distribution $N_{s} S^{2} / \theta$ is independent of the normalized flux $R$ and the coverage $\theta$. The data collapse reported in the graphs of Fig. 1 shows that the only observable effect of confinement is a small increase of $f$ for small $s / S$ when $L_{x}=2$ and $J=0.1 \mathrm{eV}$ (due to the periodic boundary conditions on the hexagonal lattice, the minimum possible value of $L_{x}$ is $L_{x}=2$ ). As a conclusion we observe that the universal scaling function $f$ is relatively insensitive to $L_{x}$, and these small variations would therefore not be easily measured in experiments.

\section{KINETIC MONTE CARLO RESULTS: ISLAND DENSITY}

However, as shown in Fig. 2(a), the total island density $N=$ $\sum_{s=2}^{\infty} N_{s}$ exhibits a strong dependence on the tube diameter. As expected, the island density $N$ is independent of $L_{x}$ for large $L_{x}$, but increases by a factor varying from 2 to 8 for small $L_{x}$. This increase is observed both for small and large $J / k_{B} T$. Note that, since the average island size $S$ is related to $N$ via the relation $S=\theta / N$, this also means that $S$ varies with $L_{x}$. A higher island density in 1D was already observed, e.g., in Fig. 2(a) of Ref. [7]. Here, we study the transition between the two limits. The origin of the higher island density in one dimension can actually be traced back to a relatively lower number of sites visited by diffusing nanoparticles in 1D [7]. Nanoparticles in 1D are therefore less efficient in finding islands to aggregate to, leading to an increased nucleation rate by collision with other nanoparticles.

Further inspection of Fig. 2(a) reveals that at large $L_{x}$ for all $\theta$ the island densities $N$ converge to the same value denoted as $N_{2 D}$ independent of $\theta$. However, the island densities split in the crossover regime. Such a result can be explained within the standard theory for submonolayer deposition, where one expects that

$$
N \sim \theta^{1-z} R^{-\chi} .
$$

It is known that $z=z_{2 D}=1$ in 2D [25] but decreases to $z=$ $z_{1 D}=3 / 4$ in $1 \mathrm{D}[10,26]$. As a consequence, $N$ should be independent of $\theta$ in 2D but depends on $\theta$ in 1D, in agreement with our results.

In order to gain more insight about the transition from 2D to 1D, we calculated the exponent $z$ from a fit of the observed variation of the density $N$ as a function of $\theta$ in the range $0.05<\theta<0.3$ for different values of $L_{x}$. Figure 2(b) shows that the variation of $z$ is continuous within the crossover. There are actually two dimensionless numbers describing the confinement: $L_{x}$ and $L_{x} N_{2 D}^{1 / 2}$, which respectively quantify confinement in terms of particle size or in terms of the typical distance between islands. The exponents $z$ seem to exhibit a better collapse when plotted as a function of $L_{x}$, suggesting that the behavior at small tube diameters is rather controlled by the particle size. However, we see in Fig. 2(c) that $z$ reaches 1 sharply for $L_{x}>L_{2 D}$, with

$$
L_{2 D} N_{2 D}^{1 / 2}=1.4 \pm 0.2 \text {. }
$$

This relation is valid both for large and small $J / k_{B} T$. Hence, we observe a purely $2 \mathrm{D}$ behavior when the tube perimeter is slightly larger than the distance between islands. Such a result is a consequence of the lack of correlation between islands beyond the first neighbors, as already suggested by the identity between capture zones and island sizes observed in Ref. [15]. At this point we notice that the interpretation of a varying $z$ is greatly simplified by the fact that the transition point at $L_{x}=L_{2 D} \sim N_{2 D}^{-1 / 2} \sim \theta^{\left(z_{2 D}-1\right) / 2} R^{\chi_{2 D} / 2}$ is independent of $\theta$ because $z_{2 D}=1$. Thus, varying $\theta$ at fixed $R$ and fixed $L_{x}$ to measure $z$, we actually never cross the transition point at 

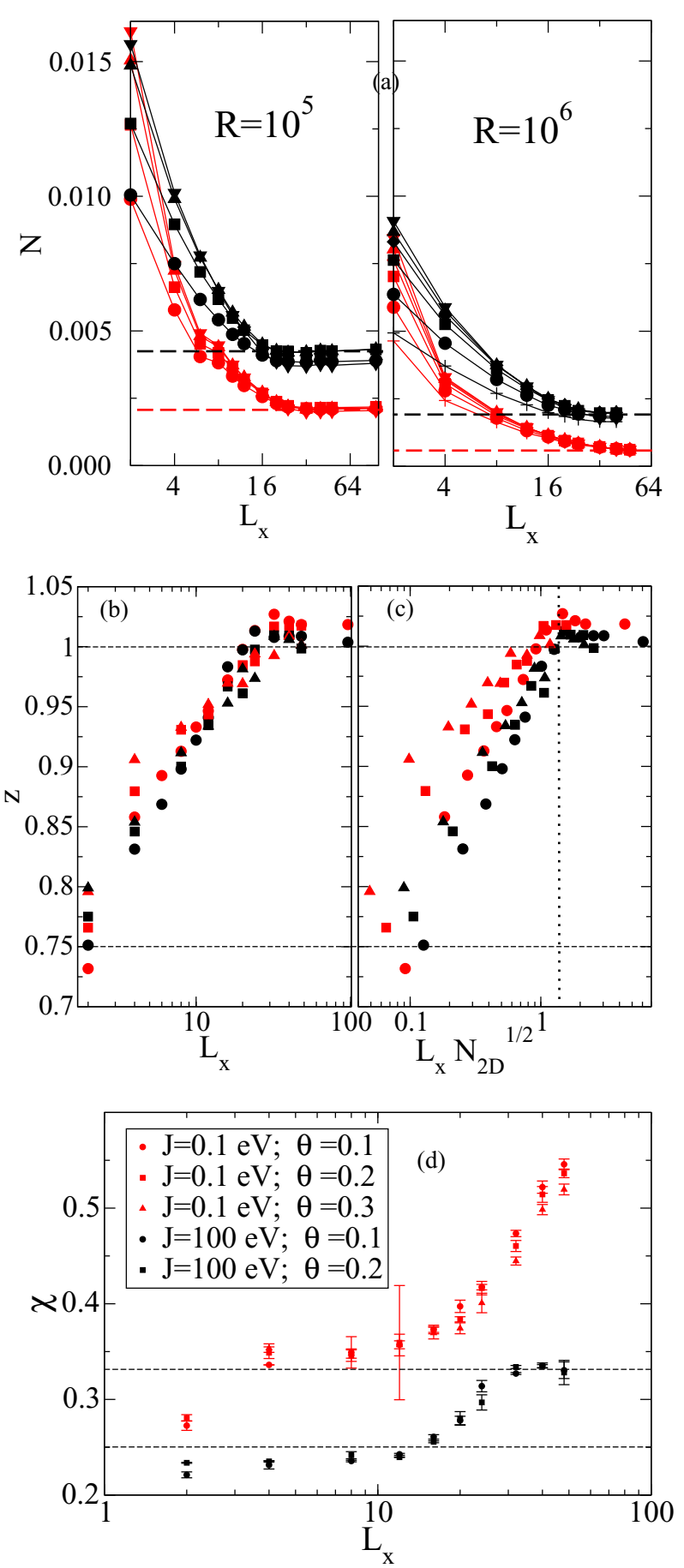

FIG. 2. (Color online) KMC simulation results. Red (gray) and black symbols correspond to the attachment-detachment regime with $J=0.1 \mathrm{eV}$ and the the irreversible aggregation regime with $J=$ $100 \mathrm{eV}$, respectively. (a) The island density $N$ increases when the tube perimeter $L_{x}$ decreases. Symbols: $+, \bullet, \mathbf{\square}, \boldsymbol{\nabla}, \boldsymbol{\nabla}$, for $\theta=$ $0.025,0.05,0.1,0.15,0.2,0.25$. $z$ exponent as a function of (b) $L_{x}$ and of (c) $L_{x} N_{2 D}^{1 / 2}$. Symbols: $\bullet, \mathbf{\square}, \boldsymbol{\Delta}$, for $R=10^{5}, 10^{5.5}$, and $10^{6}$, respectively. The $z$ exponent exhibits a transition from $z_{1 D}=3 / 4$ in $1 \mathrm{D}$ to $z_{2 D}=1 \mathrm{in} 2 \mathrm{D}$. The transition to a purely $2 \mathrm{D}$ behavior is indicated by a dotted line at $L_{x} N_{2 D}^{1 / 2} \approx 1.4 \pm 0.2$. (d) $\chi$ exponent as a function of $L_{x}$. For irreversible aggregation $J=100 \mathrm{eV}$, we find a transition from $\chi_{1 D}=1 / 4$ in $1 \mathrm{D}$ to $\chi_{2 D}=1 / 3$ in $2 \mathrm{D}$. In the attachment-detachment regime, however, there is no universal limit for $z$ in 2D.
$L_{x}=L_{2 D}$ because $L_{2 D}$ is constant. We may therefore claim that the measured exponent $z$ characterizes either the confined regime when $L_{x}<L_{2 D}$, or the $2 \mathrm{D}$ regime when $L_{x}>L_{2 D}$.

The second exponent $\chi$ is known to exhibit a universal behavior in the limit of irreversible aggregation, i.e., for large $J / k_{B} T$. We expect $\chi=\chi_{2 D}=1 / 3$ in $2 \mathrm{D}$, and $\chi=\chi_{1 D}=$ $1 / 4$ [26] in 1D. We have extracted $\chi$ from the dependence of $N$ on $R$ in KMC simulations at various $L_{x}$. Figure 2(d) clearly shows the transition for $2 \mathrm{D}$ to $1 \mathrm{D}$ at $J=100 \mathrm{eV}$. The transition of $\chi$ to its 2D limiting value is still observed for $L_{x} \sim$ $L_{2 D}$ with $L_{2 D}$ obtained from the variation of the $z$ exponent. However, the transition is blurred here because we extract $\chi$ from the $R$ dependence of $N$, and $L_{2 D} \sim N_{2 D}^{-1 / 2} \sim R^{\chi_{2 D} / 2}$ depends on $R$. Hence, for a given $L_{x}$ and close to the transition, we probe the $2 \mathrm{D}$ behavior with $L_{x}>L_{2 D}$ for small $R$ and the confined behavior with $L_{x}<L_{2 D}$ for large $R$. Due to limitations in our simulations, we cannot greatly extend the number of simulation runs and the range of $R$ to measure accurately and separately two values of $\chi$ for $L_{x}<L_{2 D}$ and for $L>L_{2 D}$. Hence, we cannot conclude about the precise behavior of $\chi$ close to the threshold. However, the existence of two plateaus with fixed values of $\chi=\chi_{1 D}$ for small $L_{x}$ and $\chi=\chi_{2 D}$ for large $L_{x}$ can be observed for any value of $\theta$ and could be consistent with a discontinuous variation of $\chi$. Further investigations are necessary in order to conclude about the continuity or the discontinuity of the $\chi$ exponent at the transition, and we hope to report along this line in the future.

As discussed by Ratsch et al. [8], the behavior of $\chi$ is less universal in the presence of detachment, i.e., for small $J / k_{B} T$. As a consequence, $\chi$ does not converge to $1 / 3$, but tends to a higher value in the $2 \mathrm{D}$ limit in Fig. 2(d). However, $\chi$ still converges to $\chi_{1 D}=1 / 4$ for small $L_{x}$ in this case.

\section{FePt NANOPARTICLE DEPOSITION ON CARBON NANOTUBES}

In order to check the predictions of the KMC simulations, we have deposited size-selected FePt nanoparticles on multiwalled carbon nanotubes (CNTs). The CNTs were obtained by chemical vapor deposition (CVD) synthesis and dispersed on a holey carbon transmission electron microscope (TEM) grid. The FePt particles, around $4 \mathrm{~nm}$ in diameter, are produced by laser vaporization and soft landed on the CNTs under ultrahigh vacuum conditions and at room temperature [27,28].

As shown in TEM images on Fig. 3(a), the incident clusters which are randomly deposited, diffuse on the tubes, and form islands [29]. Some events of a partial coalescence between neighboring particles are also observed [29]. Such a behavior is similar to that of nanoparticles deposited on graphite [see Fig. 3(b)] [30,31]. The islands exhibit a ramified shape, suggesting that the growth occurs in the irreversible aggregation regime $\left(J / k_{B} T \gg 1\right.$; see Fig. 1).

This model system allows us to compare the theoretical predictions with experimental measurements. The island density has been determined directly from TEM images, for several different CNTs with a diameter ranging from 5 to $10 \mathrm{~nm}$. Our main result, shown in Fig. 4, is the increase of the island density with decreasing nanotube diameter. This density varies by a 

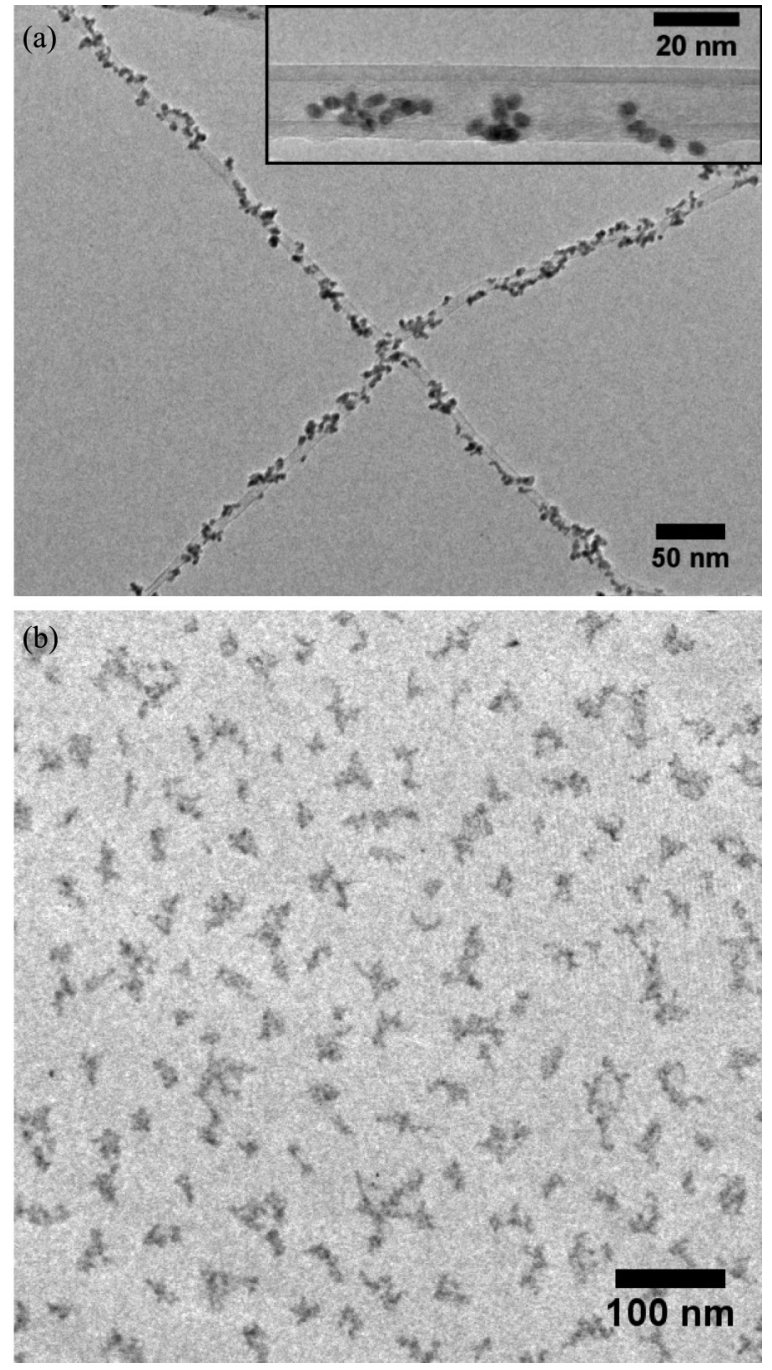

FIG. 3. Typical TEM image of $4 \mathrm{~nm}$ diameter FePt nanoparticles deposited on (a) CNTs and (b) graphite, allowing the experimental determination of island densities.

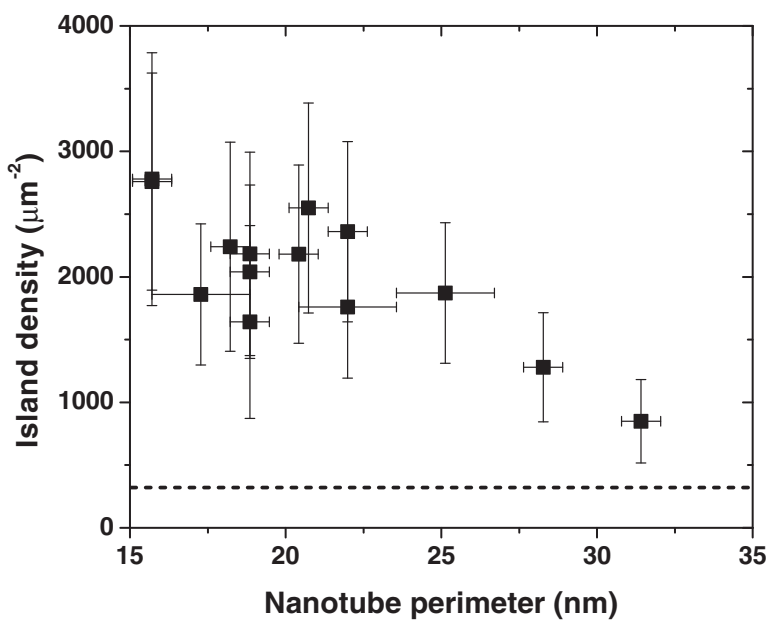

FIG. 4. Experimental nanoparticle island density as a function of CNT perimeter. The error bar on the density corresponds to the statistical uncertainty (i.e., $\sqrt{n}$ for an observation of $n$ islands). factor $\sim 3$ or 4 , in quantitative agreement with the theoretical simulations.

We did not observe the saturation of the island density for large tube diameters suggested by Fig. 2(a). Note, however, that, in contrast to our KMC simulations with homogeneous deposition on the tube, nanoparticles were experimentally deposited from a beam, and therefore always landed on the same side of the nanotube. This asymmetry should be irrelevant for small tubes and low deposition rates, when the nanoparticles have enough time to diffuse to the opposite side of the tube before meeting other nanoparticles. Instead of exploring the deposition on tubes with very large diameters where the consequences of the deposition inhomogeneity would be exacerbated, we probe the $2 \mathrm{D}$ limit with the help of a comparison to deposition on flat highly ordered pyrolytic graphite (HOPG). If the deposition were homogeneous as in the KMC simulations, the island density on a large tube $N_{2 D}$ would obey Eq. (3), and we therefore expect $N_{2 D} / N_{\mathrm{HOPG}}=\left(R_{\mathrm{HOPG}} / R_{2 D}\right)^{\chi}$, where $N_{\mathrm{HOPG}}$ is the island density on HOPG, $R_{\mathrm{HOPG}}$ is the deposition rate on $\mathrm{HOPG}$, and $R_{2 D}$ would be the homogeneous deposition flux on the tube (we have used the fact that $z=1$ for large $L_{x}$ ). The deposition on HOPG is performed at the same temperature as on the tubes, and with deposition rate two times lower. In addition we checked that nanoparticles have a sticking coefficient equal to one on nanotubes. Hence, since the ratio of the projected areas of the tube and the flat HOPG is $\pi$ and assuming that the diffusion constant of nanoparticles is the same on HOPG and on the tubes, we find $N_{2 D}=N_{\mathrm{HOPG}}(2 / \pi)^{1 / 3}$. Using this relation and the observed value $N_{\mathrm{HOPG}} \approx 372 \mu \mathrm{m}^{-2}$, we find $N_{2 D} \approx 320 \mu \mathrm{m}^{-2}$. This result is plotted in Fig. 4 and is lower than the experimentally observed values of $N$ for finite diameters, consistent with KMC simulations in Fig. 2(a).

Using the KMC simulation results (4), the transition to $2 \mathrm{D}$ behavior is expected for a tube perimeter $L_{2 D}=1.4 N_{2 D}^{-1 / 2} \approx$ $90 \mathrm{~nm}$, larger than the perimeter of the tubes observed experimentally. This confirms that experiments are performed in the transition region where a variation of the island density is expected. Note that the transition regime would be much more difficult to reach using epitaxy of atoms, for which atomic-scale tubes would be necessary. In contrast, larger clusters would not diffuse and would therefore not lead to the formation of islands. Therefore, FePt nanoscale-cluster deposition on nanotubes appears as a relevant system for the study of the 2D-to-1D transition.

\section{CONCLUSION}

To conclude, we explored the transition from 2D to $1 \mathrm{D}$ in submonolayer growth by using simulations and experiments on tubular substrates. We have presented clear evidence of an increase of the island density during submonolayer growth when the tube diameter is decreased. KMC simulations and experiments of cluster deposition on nanotubes both exhibit this effect. A detailed investigation of the simulations shows that the scaling exponents $z$ and $\chi$ indicate a transition from $2 \mathrm{D}$ to $1 \mathrm{D}$ when confinement is increased and allows us to identify the critical diameter $L_{2 D}$ below which the system is affected by confinement. Other substrates, such as top facets of graphite pleats where Ag clusters are confined, could also 
be good candidates for the observation of the dimensionality transition in submonolayer growth [22,23].

Our work opens several perspectives. First, growth on single-wall nanotubes (SWNT) could also be performed. Due to their softness, SWNTs lead to stronger elastic interactions between nanoparticles, and may therefore trigger complex periodic organizations of nanoparticles, as recently discussed in Ref. [32]. In addition, the existence of a dimensionality transition on tubular substrates open novel questions for the studies of island size and capture zone distributions in submonolayer

[1] A. Barabási and H. Stanley, Fractal Concepts in Surface Growth (Cambridge University Press, Cambridge, 1996).

[2] J. Kim, Nature (London) 458, 740 (2009).

[3] R. Ruiz, B. Nickel, N. Koch, L. C. Feldman, R. F. Haglund, A. Kahn, F. Family, and G. Scoles, Phys. Rev. Lett. 91, 136102 (2003).

[4] S. A. Burke, J. M. Mativetsky, R. Hoffmann, and P. Grütter, Phys. Rev. Lett. 94, 096102 (2005).

[5] F. Rabbering, A. Kara, H. Wormeester, T. Warnaar, O. Trushin, T. S. Rahman, and B. Poelsema, Phys. Rev. Lett. 103, 096105 (2009).

[6] Y. Shim and J. G. Amar, Phys. Rev. Lett. 108, 076102 (2012).

[7] M. C. Bartelt and J. W. Evans, Phys. Rev. B 46, 12675 (1992).

[8] C. Ratsch, A. Zangwill, P. Šmilauer, and D. D. Vvedensky, Phys. Rev. Lett. 72, 3194 (1994).

[9] J. Villain and A. Pimpinelli, Physique de la Croissance Cristalline (Eyrolles, Paris, 1995).

[10] J. G. Amar, M. N. Popescu, and F. Family, Phys. Rev. Lett. 86, 3092 (2001).

[11] T. Michely and J. Krug, Islands, Mounds, and Atoms (Springer, Berlin, 2004).

[12] M. Einax, S. Ziehm, W. Dieterich, and P. Maass, Phys. Rev. Lett. 99, 016106 (2007).

[13] A. Pimpinelli and T. L. Einstein, Phys. Rev. Lett. 99, 226102 (2007).

[14] T. J. Oliveira and F. D. A. Aarão Reis, Phys. Rev. B 86, 115402 (2012).

[15] M. Fanfoni, F. Arciprete, C. Tirabassi, D. Del Gaudio, A. Filabozzi, A. Balzarotti, F. Patella, and E. Placidi, Phys. Rev. E 86, 061605 (2012).

[16] M. Körner, M. Einax, and P. Maass, Phys. Rev. B 86, 085403 (2012).

[17] P. Gambardella, M. Blanc, H. Brune, K. Kuhnke, and K. Kern, Phys. Rev. B 61, 2254 (2000). growth [13-16]. Finally, our studies help to understand the morphology of cluster-CNT hybrid structures, which could be technologically relevant, e.g., for biosensors [33], drug delivery [34], or magnetic force microscopy (MFM) [35].

\section{ACKNOWLEDGMENT}

We thank one of the referees for important comments which helped to improve the paper.

[18] S. Rusponi, T. Cren, N. Weiss, M. Epple, P. Buluschek, L. Claude, and H. Brune, Nat. Mater. 2, 546 (2003).

[19] T. J. Stasevich, C. Tao, W. G. Cullen, E. D. Williams, and T. L. Einstein, Phys. Rev. Lett. 102, 085501 (2009).

[20] A. Ramadan, F. Picaud, and C. Ramseyer, Surf. Sci. 604, 1576 (2010).

[21] G. Grochola, I. K. Snook, and S. P. Russo, Surf. Sci. 606, 1565 (2012).

[22] M. Schmidt, N. Kébaili, A. Lando, S. Benrezzak, L. Baraton, P. Cahuzac, A. Masson, and C. Bréchignac, Phys. Rev. B 77, 205420 (2008).

[23] A. F. Kemper, H.-P. Cheng, N. Kébaïli, S. Benrezzak, M. Schmidt, A. Masson, and C. Bréchignac, Phys. Rev. B 79, 193403 (2009).

[24] G. Bales, Surf. Sci. 356, L439 (1996).

[25] J. G. Amar, F. Family, and P.-M. Lam, Phys. Rev. B 50, 8781 (1994).

[26] J. A. Blackman and P. A. Mulheran, Phys. Rev. B 54, 11681 (1996).

[27] A. Perez et al., Int. J. Nanotechnol. 7, 523 (2010).

[28] D. Tainoff, L. Bardotti, F. Tournus, G. Guiraud, O. Boisron, and P. Mélinon, J. Phys. Chem. C 112, 6842 (2008).

[29] L. Bardotti et al. (unpublished).

[30] F. Tournus, L. Bardotti, and V. Dupuis, J. Appl. Phys. 109, 114309 (2011).

[31] L. Bardotti, F. Tournus, M. Pellarin, M. Broyer, P. Mélinon, and V. Dupuis, Surf. Sci. 606, 110 (2012).

[32] J. C. Pàmies and A. Cacciuto, Phys. Rev. Lett. 106, 045702 (2011).

[33] V. Georgakilas, D. Gournis, V. Tzitzios, L. Pasquato, D. M. Guldi, and M. Prato, J. Mater. Chem. 17, 2679 (2007).

[34] S. Utsumi, K. Urita, H. Kanoh, M. Yudasaka, K. Suenaga, S. Iijima, and K. Kaneko, J. Phys. Chem. B 110, 7165 (2006).

[35] Z. Deng, E. Yenilmez, J. Leu, J. E. Hoffman, E. W. J. Straver, H. Dai, and K. A. Moler, Appl. Phys. Lett. 85, 6263 (2004). 